\title{
IS THE BIOLOGIC STANDARD OF SQUILL AND THE PREPARATIONS THEREOF CORRECT?
}

BY H. C. COLSON, JR., AND H. ENGELHARDT.

Both fluidextract and tincture of squill when prepared from a selected drug strictly according to the official process very frequently fail to meet the biologic requirements of the U.S. P. IX. This may be due to the manufacturing process in which incomplete extraction of the active principle may occur and to an incorrect biologic standard adopted by the Pharmacopoeia.

The Pharmacopoeia requires that the two preparations be of the same biologic strength as the fluidextract and tincture of digitalis, in other words, that $0.006 \mathrm{Gm}$. of both fluidextract of digitalis and fluidextract of squill and 0.0006 $\mathrm{Gm}$. of tincture of digitalis and tincture of squill be the minimum systolic (not lethal) dose for each gramme of body weight of frog; but while the dose for fluidextract of digitalis is given with 0.05 mil and that for the tincture with $0.5 \mathrm{mil}$, o. I mil or double the dose is given as the average dose of fluidextract of squill and also $\mathrm{I}$.o mil for the tincture. Thus it may be justly assumed that the Pharmacopoeia admits that squill preparations possess only half the strength of the corresponding digitalis preparations. This assumption seems to be correct as can be seen from the following results, which were based on the requirements for the fluidextract that 0.0006 be the minimum systolic dose per gramme of body weight of frog and 0.006 be the corresponding dose for the tincture.

In order to test the biologic strength of various commercial fluidextracts of squill four samples of this preparation made by as many different manufacturers and designated as I, II, III, IV, were examined by the cat method, the one-hour frog and the 12 -hour frog methods. The following results expressed in percentage of the official standard were obtained:

\begin{tabular}{|c|c|c|c|}
\hline I. . & $\begin{array}{l}\text { Cat Method. } \\
42.2 \text { percent }\end{array}$ & $\begin{array}{c}\text { 1-Hour Frog Method. } \\
53.3 \text { percent }\end{array}$ & $\begin{array}{l}\text { 12-Hour Frog Method. } \\
42.2 \text { percent }\end{array}$ \\
\hline II. & 25.4 percent & 63.2 percent & 33.8 percent \\
\hline III. & 68.5 percent & 200.0 percent & 120.5 percent \\
\hline$\ldots \ldots \ldots \ldots \ldots \ldots \ldots$ & 43.7 percent & I 44.0 percent & 52.8 percent \\
\hline
\end{tabular}

The results obtained by the cat method were based upon a standard of roo milligrammes of squill for kilo body weight of cat, which corresponds to the standard of fluidextract of digitalis.

The alcohol contents of the different preparations varied from 37 percent to 55 percent; the fluidextracts with a low percentage appeared somewhat viscid while those with a high percentage were rather mobile.

It is our intention to continue the experiments with both the fluidextract and tincture of squill and we hope to report in the near future on the merits of the different assay methods and the value of the official manufacturing process.

RESEARCh Laboratory OF SHaRp \& DOHME, BaLtimorf. 